\title{
Hormonal contraception and the risk of severe asthma exacerbation: 17-year population-based cohort study
}

\author{
Bright I Nwaru (D) , ${ }^{1,2}$ Holly Tibble, ${ }^{3}$ Syed A Shah, ${ }^{3}$ Rebecca Pillinger, ${ }^{3}$ \\ Susannah McLean, ${ }^{3}$ Dermot P Ryan, ${ }^{3,4}$ Hilary Critchley, ${ }^{5}$ David B Price (D) , 4,6,7 \\ Catherine M Hawrylowicz, ${ }^{8}$ Colin R Simpson, ${ }^{3,9}$ Ireneous N Soyiri, ${ }^{3,10}$ \\ Francis Appiagyei, ${ }^{4}$ Aziz Sheikh ${ }^{3}$
}

- Additional material is published online only. To view, please visit the journal online (http://dx.doi.org/10.1136/ thoraxjnl-2020-215540).

For numbered affiliations see end of article.

\section{Correspondence to}

Dr Bright I Nwaru, Krefting Research Centre, University of Gothenburg, Goteborg SE-405 30, Sweden;

bright.nwaru@gu.se

Received 12 June 2020

Revised 21 September 2020

Accepted 24 September 2020

Published Online First

23 November 2020

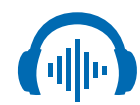

Listen to Podcast thorax.bmj.com

Check for updates

(c) Author(s) (or their employer(s)) 2021. No commercial re-use. See rights and permissions. Published by BMJ.

To cite: Nwaru Bl, Tibble H, Shah SA, et al. Thorax 2021;76:109-115.

\begin{abstract}
Background Longitudinal studies investigating impact of exogenous sex steroids on clinical outcomes of asthma in women are lacking. We investigated the association between use of hormonal contraceptives and risk of severe asthma exacerbation in reproductive-age women with asthma.

Methods We used the Optimum Patient Care Research Database, a population-based, longitudinal, anonymised primary care database in the UK, to construct a 17-year (1 January 2000-31 December 2016) retrospective cohort of reproductive-age ( $16-45$ years, $n=83084$ ) women with asthma. Using Read codes, we defined use, subtypes and duration of use of hormonal contraceptives. Severe asthma exacerbation was defined according to recommendations of the European Respiratory Society/American Thoracic Society as asthma-related hospitalisation, accident and emergency department visits due to asthma and/or oral corticosteroid prescriptions. Analyses were done using multilevel mixed-effects Poisson regression with QR decomposition.
\end{abstract}

Results The 17-year follow-up resulted in 456803 person-years of follow-up time. At baseline, $34 \%$ of women were using any hormonal contraceptives, 25\% combined (oestrogen/progestogen) and 9\% progestogen-only contraceptives. Previous (incidence rate ratio (IRR) 0.94, $95 \% \mathrm{Cl} 0.92$ to 0.97 ) and current (IRR $0.96,95 \% \mathrm{Cl} 0.94$ to 0.98 ) use of any, previous (IRR $0.92,95 \% \mathrm{Cl} 0.87$ to 0.97 ) and current use of combined (IRR $0.93,95 \% \mathrm{Cl} 0.91$ to 0.96 ) and longer duration of use (3-4 years: IRR 0.94, $95 \% \mathrm{Cl} 0.92$ to $0.97 ; 5+$ years: IRR $0.91,95 \% \mathrm{Cl} 0.89$ to 0.93) of hormonal contraceptives, but not progestogen-only contraceptives, were associated with reduced risk of severe asthma exacerbation compared with non-use.

Conclusions Use of hormonal contraceptives may reduce the risk of severe asthma exacerbation in reproductive-age women. Mechanistic studies investigating the biological basis for the influence of hormonal contraceptives on clinical outcomes of asthma in women are required. Protocol registration number European Union electronic Register of Post-Authorisation Studies (EUPAS22967).

\section{INTRODUCTION}

The female sex steroid hormones are thought to partly explain the notable sex-related differences

\section{Key messages}

What is the key question?

- Does the use of hormonal contraceptives have any impact on severe asthma exacerbation in reproductive-age women?

What is the bottom line?

- Use of hormonal contraceptives was associated with a small protective effect on severe asthma exacerbations in reproductive-age women.

Why read on?

- This is the largest longitudinal study investigating the impact of hormonal contraceptives on a key clinical outcome of asthma in reproductive-age women with already established asthma.

in incidence and manifestation of clinical outcomes of asthma. ${ }^{12}$ Fluctuations of the levels of estradiol and progesterone during women's menstrual cycle have been linked to worsening of asthma symptoms in some women. ${ }^{3}$ A predominance of T-helper (Th) cell two over Th cell 1-mediated immunity has also been observed during the premenstrual period. ${ }^{45}$ It is estimated that up to $45 \%$ of women with asthma experience heightened exacerbation during the premenstrual period. ${ }^{6}$ In some women, the use of exogenous hormonal contraceptives appears to improve asthma outcomes, including reduced Th2 responses, reduced asthma symptoms and improved lung function..$^{7-10}$ In a previous exploratory analysis of data from serial national cross-sectional Scottish Health Surveys, we observed up to 32\% (95\% CI 2 to 53 ) reduction in asthma exacerbations and up to $55 \%$ (95\% CI 18 to 75 ) reduction in asthma hospital episodes in reproductive age females using hormonal contraceptives compared with those not using exogenous sex hormones. ${ }^{11}$

Although the role of exogenous sex steroids in asthma in women has been the subject of intense investigations for several decades, findings remain conflicting and inconclusive. Our recent systematic review and meta-analysis highlighted the lack of prospective studies investigating the impact of use of hormonal contraceptives on clinical outcomes 
of asthma in women with established asthma. ${ }^{12}$ In previous studies, the association of use of hormonal contraceptives with asthma outcomes has typically been studied by including women with and without asthma. ${ }^{12}$ Such studies, the majority of which have been cross-sectional, are at high risk of bias, including selfselection bias, patient reporting bias, lack of objective measures of exposures and outcomes and, in particular, confounding bias. ${ }^{12}$ A more robust design is to undertake long-term follow-up of a cohort of women with already established asthma and investigate the influence of use of hormonal contraceptives on subsequent clinical outcomes. Deciphering the role of hormonal contraceptives on clinical outcomes of asthma is essential in resolving the current uncertainty in the underlying evidence. This is also essential in evaluating any potential benefit or harm of exogenous sex steroids in the management of asthma.

In the current study, we investigated the association of use of hormonal contraceptives, their subtypes and duration of use with severe asthma exacerbation in reproductive-age women. We used the Optimum Patient Care Research Database (OPCRD), ${ }^{13}{ }^{14} \mathrm{a}$ large population-based, longitudinal, anonymised primary care database in the UK, to construct a cohort of reproductive-age (16-45 years) women with asthma. We also assessed whether body mass index (BMI) and cigarette smoking modified these associations.

\section{METHODS}

\section{Ethics approvals and permissions}

The Anonymised Data Ethics and Protocol Transparency Committee, responsible for approval of research using the OPCRD database, gave approval for the current study (Ref. number: ADEPT1317). In addition, Optimum Patient Care has an existing ethics approval for the use of OPCRD for research purposed from the National Health Service (NHS) Health Research Authority (REC Ref: 15/EM/150). All researchers involved in the analysis of the data completed required information governance courses.

\section{Protocol registration and publication}

We registered the study protocol with the European Union electronic Register of Post-Authorisation Studies (EUPAS22967), which was additionally published in a peer-reviewed journal prior to undertaking the analyses. ${ }^{15}$

\section{Study design and population}

OPCRD is a longitudinal, anonymised primary care database, comprising 630 primary care practices across the UK with over 6 million patients at the time of extracting data for the current study. The database is used to conduct epidemiological, clinical and pharmaceutical studies (http://optimumpatientcare.org/opcrd/). ${ }^{13}$ We established an open retrospective cohort of reproductive-age women aged 16-45 years who had any asthma event (including diagnosis, hospitalisation, medication prescription) using previously established Read codes (online supplemental file 1) starting from baseline on 1 January 2000 or 5 years prior to this date. Participants entered the cohort from baseline or date of registration with a general practice or the year of turning age 16 years and were followed-up until 31 December 2016. Participants exited the cohort on the date of death, deregistration from a practice, year of turning 45 years, or 31 December 2016, whichever came first. Women were excluded in any year they were pregnant and included in the years they were not pregnant. In total, the study cohort comprised 83084

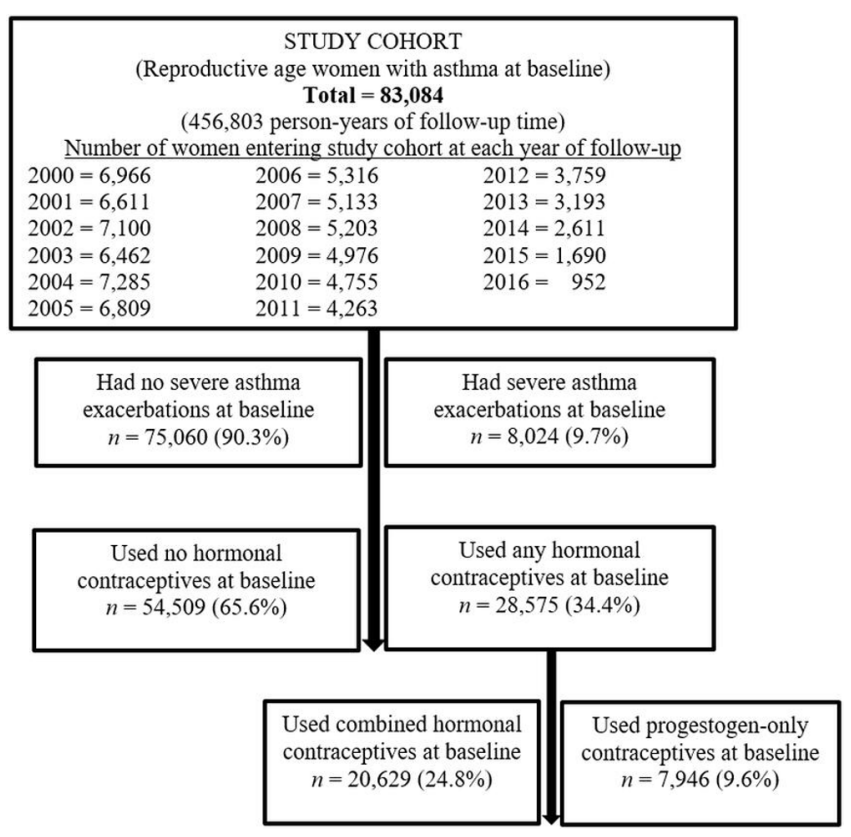

Figure 1 Flowchart of study cohort and baseline severe asthma exacerbations and use of hormonal contraceptives

women with a total of 456803 person-years of follow-up time over the 17-year period (figure 1).

\section{Ascertainment of hormonal contraceptives}

We used the Read Clinical Classification System ${ }^{16}$ (Read codes; online supplemental file 2) to extract information on prescription of hormonal contraceptives. Based on the extracted information, we defined the following exposures for the use of hormonal contraceptives for each year of follow-up:

1. Previous (any time in the past) and current (during that year) use vs non-use

2. Previous and current use of subtypes of hormonal contraceptives (combined oestrogen/progestogen, progestogen-only vs non-use)

3. Duration of use: 1-2 years, 3-4 years, 5 years or more vs non-use.

In each instance, we counted use of each of hormonal contraceptives only if it occurred before a record of severe asthma exacerbation.

\section{Potential confounding variables}

We selected potential confounding variables for adjustment in the analysis based on evidence from the previous literature on the topic and on the basis of the evidence provided in our data. We used relevant Read codes (online supplemental file 3) to extract data from the database for the following variables: gravidity, BMI, smoking, Charlson Comorbidity Index, ${ }^{17}$ gynaecological conditions (endometriosis, polycystic ovary syndrome, acne, bilateral salpingo-oophorectomy (BSO), hysterectomy with $\mathrm{BSO}$, hysterectomy without BSO, fibroids, and menstrual bleeding complaints identified under coding terms: menorrhagia, metrorrhagia menometrorrhagia) ${ }^{18}$ and Index of Multiple Deprivation (IMD). ${ }^{19}$ Age was derived using the date of birth. Our a priori plan was to carry out an analysis using propensity scores. ${ }^{15}$ However, we were unable to design a suitable statistical model (within the context of time-varying, multilevel, mixed-effects, negative binomial or 
Poisson regression models) and instead performed a conventional confounding adjustment.

\section{Study outcome}

The study outcome was severe asthma exacerbations (Read codes in online supplemental file 1), defined according to the recommendation of the European Respiratory Society/American Thoracic Society ${ }^{20}$ as asthma-related hospitalisation, accident and emergency department visits due to asthma and/or prescription for oral corticosteroids. Any exacerbations occurring within a 14-day period were considered as one event. This definition has been validated and used in previous studies within the OPCRD database. ${ }^{21}$ Read codes used to define the study outcome are available in online supplemental file 1 .

\section{Statistical analyses}

The Pearson's Chi-square test and differences in means were used to describe the distribution of the baseline study characteristics in relation to the use of hormonal contraceptives and the study outcomes. We initially used multilevel, mixed-effects, negative binomial regression to study the associations between use of hormonal contraceptives and severe asthma exacerbations, but this resulted in non-convergence of some of the models, particularly the models for smoking-stratified analyses. To improve the convergence of the models, we used multilevel, mixed effects Poisson regression with QR decomposition, which provides an alternative approach to fitting models for Poisson distribution data by using the QR decomposition of the variance-components matrix. The approach helps to improve the convergence of models when the variance components is close to the border of the parameter space. $^{22}$ The models accounted for variations across general practices and the longitudinal nature of the variables. We adjusted for the confounding variables described in the previous section and the variables were included in the models using the categories given in table 1 . All variables were treated as time-varying in the models, so that each variable was allowed to take different values at each year of follow-up. We evaluated interactions of BMI and smoking with hormonal contraceptives by including interaction terms in each adjusted model; the analyses were subsequently stratified by BMI or smoking only if the interaction terms achieved $p$ values of $<0.20$. As both BMI and smoking were analysed as time-varying variables, we assumed that their interactions with the use of hormonal contraceptives in relation to the risk of severe asthma exacerbations were constant over the study follow-up time. We evaluated the potential for residual confounding after the adjusted regression analyses by calculating the E-values for the observed estimates of association of use of hormonal contraceptives and severe asthma exacerbations. ${ }^{23}$ The derived estimates of the E-value signify the minimum magnitude of association that an unmeasured confounder should have both in relation to respective exposures and the outcome in order to negate the observed estimate of association between the exposures and the outcome. ${ }^{23}$

Data management and editing were undertaken using $\mathrm{R}$ version 3.5.1 (R Foundation for Statistical Computing, Vienna, Austria). Statistical analyses were undertaken using and Stata 14 (Stata Statistical Software: Release 14; StataCorp LP, College Station, TX, USA).

\section{Reporting}

This article is reported following the recommendations of STROBE (Strengthening the Reporting of Observational Studies in Epidemiology) ${ }^{24}$ and RECORD (Reporting of studies Conducted using Observational Routinely-collected Data). ${ }^{25}$

\section{RESULTS}

\section{Characteristics of the study populations}

Table 1 shows the characteristics of the study population. At baseline, $34 \%$ of the women were using any hormonal contraceptives: 25\% combined (oestrogen/progestogen) and 9\% progestogen-only. Women aged between 21 and 25 years were more likely to use any combined hormonal contraceptives than women of other age groups, but after this age the use of hormonal contraceptives decreased with increasing age. Those who had smoked or were currently smoking were less likely to use combined hormonal contraceptives, but were more likely to use progestogen-only contraceptives. Use of any combined hormonal contraceptives decreased with increasing BMI and number of previous pregnancies, but use of progestogen-only contraceptives increased with increasing BMI and number of previous pregnancies. Use of combined hormonal contraceptives was lower but use of progestogen-only contraceptives was higher in those with at least one gynaecological condition than in those without. Use of any combined hormonal contraceptives was lower, but use of progestogen-only contraceptives was higher in those with at least one Charlson Comorbidity Index score than in those with zero score. The proportion of women with severe asthma exacerbation at baseline increased with increasing age; was higher in ever-smokers than in non-smokers; increased with increasing BMI; increased with increasing number of previous pregnancies; was higher in women with a prior gynaecological condition than those without; was higher in women with any comorbidity than in women without any comorbidity; and was relatively equally distributed between IMD quintiles (table 1).

\section{Association between use of hormonal contraceptives and asthma exacerbations}

The unadjusted and adjusted associations between use of hormonal contraceptives and severe asthma exacerbations are shown in figure 1. In the adjusted models, previous and current use of any, combined, and longer duration of use were associated with a reduced risk of severe asthma exacerbations compared with non-use. While women using hormonal contraceptives for 1-2 years had a null risk for asthma exacerbations, those using it for 3-4 years and those using ir for $\geq 5$ years were at a reduced risk compared with non-users (figure 2). When we stratified the analyses by BMI and smoking, the risk estimates did not substantially differ across BMI and smoking strata (table 2). However, while the BMI-stratified results were statistically significant for combined oestrogen/progestogen contraceptives across the BMI strata, this was not the case for progestogen-only contraceptives (table 2). We further separately analysed the category of very lean women (ie, those with BMI $<20 \mathrm{~kg} / \mathrm{m}^{2}$ ) but we did not find the observed protection among these women, instead there was slight non-significant increased risk of severe asthma exacerbation (data not shown). The E-values for the association between hormonal contraceptives and asthma exacerbations ranged from 1.00 to 1.39 . These values signify the magnitude of risk estimates required by an unmeasured confounder, beyond the adjusted confounders, to negate the observed risk estimates. For example, an unmeasured confounder requires a minimum risk ratio of 1.00 and maximum of 1.39 in order to negate the observed association between hormonal contraceptives and asthma exacerbation after adjusting for all other confounding factors.

\section{DISCUSSION}

In this large, population-based, longitudinal cohort study of reproductive-age women followed for 17 years, we found that 
Table 1 Baseline characteristics by use of hormonal contraceptives in reproductive-age (16-45 years) women with asthma and mean number of severe asthma exacerbations

\begin{tabular}{|c|c|c|c|c|c|}
\hline Background characteristic & $\begin{array}{l}\text { Frequency } \\
(\mathrm{n}=83084) \\
\mathrm{n}(\%)\end{array}$ & $\begin{array}{l}\text { Used any hormonal } \\
\text { contraceptives }(n=28 \\
575,34 \%) \\
\%(95 \% \mathrm{Cl})^{*}\end{array}$ & $\begin{array}{l}\text { Used combined hormonal } \\
\text { contraceptives ( } n=20 \\
629,25 \%) \\
\%(95 \% \mathrm{Cl})^{*}\end{array}$ & $\begin{array}{l}\text { Used progesterone-only } \\
\text { hormonal contraceptives } \\
(\mathrm{n}=7946,9 \%) \\
\%(95 \% \mathrm{Cl})^{*}\end{array}$ & $\begin{array}{l}\text { Severe asthma } \\
\text { exacerbations at baseline } \\
(\mathrm{n}=8024,10 \%) \\
\%(95 \% \mathrm{Cl})^{*}\end{array}$ \\
\hline \multicolumn{6}{|l|}{ Age (years) } \\
\hline $16-20$ & $24504(29.5)$ & 42.6 (41.9 to 43.2$)$ & $35.3(34.7$ to 35.9$)$ & $7.2(6.9$ to 7.5$)$ & $8.3(8.0$ to 8.7$)$ \\
\hline $21-25$ & $11754(14.1)$ & 47.4 (46.5 to 48.3 ) & 36.7 (35.8 to 37.6$)$ & 10.7 (10.2 to 11.3$)$ & 9.1 (8.6 to 9.6 ) \\
\hline $26-30$ & $13870(16.7)$ & 38.5 (37.7 to 39.3$)$ & 26.9 (26.2 to 27.7$)$ & 11.5 (11.0 to 12.1$)$ & $10.0(9.5$ to 10.5$)$ \\
\hline $31-35$ & $14985(18.0)$ & 28.0 (27.3 to 28.7$)$ & 16.7 (16.1 to 17.3$)$ & 11.3 (10.8 to 11.8$)$ & 10.3 (9.8 to 10.8$)$ \\
\hline $36-40$ & $12456(15.0)$ & $18.8(18.2$ to 19.5$)$ & 9.3 (8.8 to 9.8 ) & 9.6 (9.1 to 10.1$)$ & 10.6 (10.0 to 11.1$)$ \\
\hline $41-45$ & $5515(6.6)$ & 12.5 (11.7 to 13.4$)$ & 4.7 (4.2 to 5.3$)$ & 7.8 (7.1 to 8.6$)$ & 12.1 (11.2 to 13.0$)$ \\
\hline \multicolumn{6}{|l|}{ Smoking status } \\
\hline Non-smokers & $39982(48.1)$ & 34.6 (34.1 to 35.1$)$ & 26.2 (25.7 to 26.6 ) & $8.4(8.1$ to 8.7$)$ & 8.0 (7.8 to 8.3$)$ \\
\hline Ever smokers & $43102(51.9)$ & 34.2 (33.7 to 34.6$)$ & 23.6 (23.2 to 24.0$)$ & $10.6(10.3$ to 10.9$)$ & 11.1 (10.9 to 11.4$)$ \\
\hline \multicolumn{6}{|l|}{ Body mass index $\left(\mathrm{kg} / \mathrm{m}^{2}\right)$} \\
\hline$<25$ & $40018(48.2)$ & 37.8 (37.3 to 38.3$)$ & $29.9(29.5$ to 30.3$)$ & $7.9(7.6$ to 8.1$)$ & 8.0 (7.7 to 8.3$)$ \\
\hline $25-29.9$ & $21555(25,9)$ & 35.1 (34.4 to 35.7 ) & 25.5 (24.9 to 26.0$)$ & $9.6(9.2$ to 10.0$)$ & 9.5 (9.2 to 10.0$)$ \\
\hline$\geq 30$ & $21511(25,9)$ & $27.4(26.8$ to 28.0$)$ & 14.7 (14.2 to 15.2$)$ & $12.7(12.2$ to 13.1$)$ & $12.8(12.4$ to 13.3$)$ \\
\hline \multicolumn{6}{|l|}{ Gravidity } \\
\hline 0 & $50772(61.1)$ & 34.9 (34.5 to 35.3$)$ & 27.7 (27.3 to 28.1$)$ & $7.2(7.0$ to 7.4$)$ & 8.7 (8.4 to 8.9$)$ \\
\hline 1 & $15319(18.4)$ & 36.5 (35.7 to 37.2 ) & 24.7 (24.0 to 25.4$)$ & 11.8 (11.3 to 12.3$)$ & 10.1 (9.6 to 10.6$)$ \\
\hline 2 & $9560(11.5)$ & $33.2(32.2$ to 34.1$)$ & 18.7 (18.0 to 19.5$)$ & 14.4 (13.7 to 15.2$)$ & $11.3(10.7$ to 12.0$)$ \\
\hline $3+$ & $7433(9.0)$ & $28.2(27.2$ to 29.2$)$ & 13.5 (12.8 to 14.3$)$ & 14.7 (13.9 to 15.5$)$ & 13.1 (12.4 to 13.9$)$ \\
\hline \multicolumn{6}{|l|}{ Any gynaecological condition $\dagger$} \\
\hline No & $62729(75.5)$ & 34.0 (33.7 to 34.4$)$ & 25.5 (25.1 to 25.8$)$ & 8.5 (8.3 to 8.8$)$ & $8.6(8.4$ to 8.9$)$ \\
\hline Yes & $20355(24.5)$ & 35.5 (34.8 to 36.1$)$ & 22.8 (22.2 to 23.4$)$ & $12.7(12.2$ to 13.1$)$ & $12.7(12.3$ to 13.2$)$ \\
\hline \multicolumn{6}{|l|}{ Charlson Comorbidity Index } \\
\hline 0 & $78.749(94.8)$ & 34.8 (34.5 to 35.1$)$ & 25.3 (25.0 to 25.6$)$ & 9.4 (9.3 to 9.7$)$ & 9.3 (9.1 to 9.5$)$ \\
\hline 1 & $4335(5.2)$ & 26.8 (25.5 to 28.2$)$ & $15.8(14.7$ to 16.9$)$ & $11.0(10.1$ to 12.0$)$ & 15.7 (14.6 to 16.8$)$ \\
\hline \multicolumn{6}{|c|}{ Index of Multiple Deprivation quintiles } \\
\hline First quintile (least deprived) & $16968(20.4)$ & 28.8 (28.1 to 29.5$)$ & 21.0 (20.4 to 21.6$)$ & $7.7(7.3$ to 8.1$)$ & 9.1 (8.7 to 9.6$)$ \\
\hline Second quintile & $15732(18.9)$ & 34.0 (33.2 to 34.7$)$ & 24.7 (24.1 to 25.4$)$ & 9.2 (8.8 to 9.7$)$ & $10.6(10.2$ to 11.1$)$ \\
\hline Third quintile & $16554(19.9)$ & 36.6 (35.9 to 37.4$)$ & 26.2 (25.5 to 26.9$)$ & $10.4(9.9$ to 10.9$)$ & 9.0 (8.6 to 9.5$)$ \\
\hline Fourth quintile & $17004(20.5)$ & $35.2(34.5$ to 35.9$)$ & 25.3 (24.6 to 26.0$)$ & 9.9 (9.5 to 10.4$)$ & $10.5(10.1$ to 11.0$)$ \\
\hline Fifth quintile (most deprived) & $16826(20.2)$ & 37.4 (36.7 to 38.1 ) & 26.8 (26.2 to 27.5$)$ & 10.5 (10.1 to 11.0$)$ & 9.0 (8.6 to 9.4$)$ \\
\hline
\end{tabular}

${ }^{*}$ Difference between groups of background variables were statistically significant $(p<0.001)$.

tDefined as having any of the following conditions: endometriosis, polycystic ovary syndrome, menorrhagia, acne, metrorrhagia, bilateral salpingo-oophorectomy (BSO), hysterectomy BSO, hysterectomy, fibroids and menometrorrhagia.

previous and current use of any, combined and longer duration of use of hormonal contraceptives were associated with a reduced, but small risk, of severe asthma exacerbations, compared with non-use. Sensitivity analyses evaluating the potential for residual confounding explaining these results indicate that we cannot exclude the impact of uncontrolled confounding factors from partly explaining our findings.

The OPCRD cohort is well characterised and largely representative of the UK primary care population. At the time of the current study, the database comprised 6.3 million patients from 630 general practices derived from across the UK. With large sample sizes, we could study the role of both duration and subtypes of hormonal contraceptives, thereby enabling good precision in estimates of effect sizes. The 17-year longitudinal follow-up provided sufficient time to study the associations of use of hormonal contraceptives with asthma exacerbations and allowed investigation of the time-dependence of the exposures. The use of the Read Clinical Classification System for assessment of all study variables ensured consistent recording of the study variables. ${ }^{15}$ The UK national initiative, Quality and Outcomes Framework (QOF), which gave incentives to primary care to maintain a register of asthma patients, was established in 2004, leading to noticeable improvements in recording of clinical events of asthma. Thirteen of the 17 years of follow-up of our study occurred post-adoption of QOF, which should minimise bias resulting from underdiagnosis of asthma. ${ }^{26}$ The definition of the study outcome, severe asthma exacerbation, followed the recommended definition of the European Respiratory Society/American Thoracic Society ${ }^{20}$ and has been validly ascertained within the OPCRD database. ${ }^{21}$ To minimise the risk 


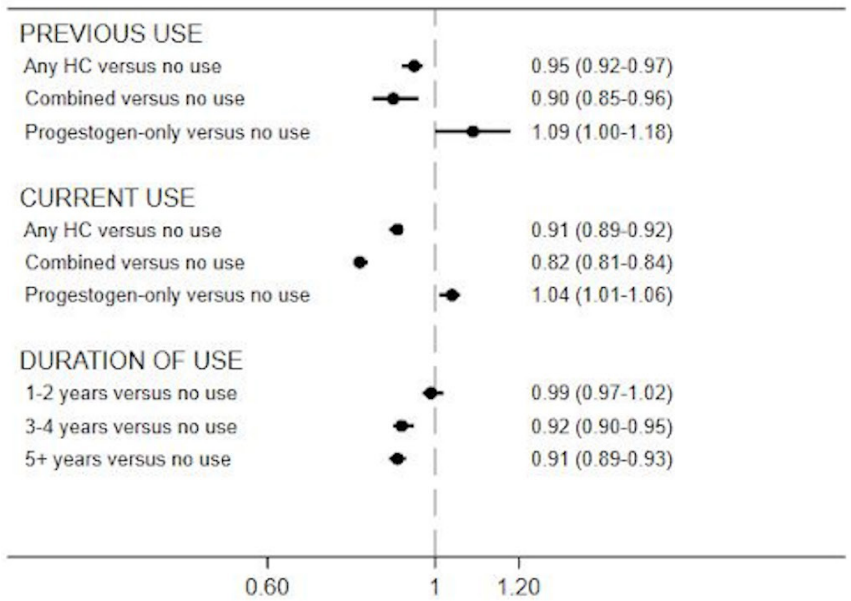

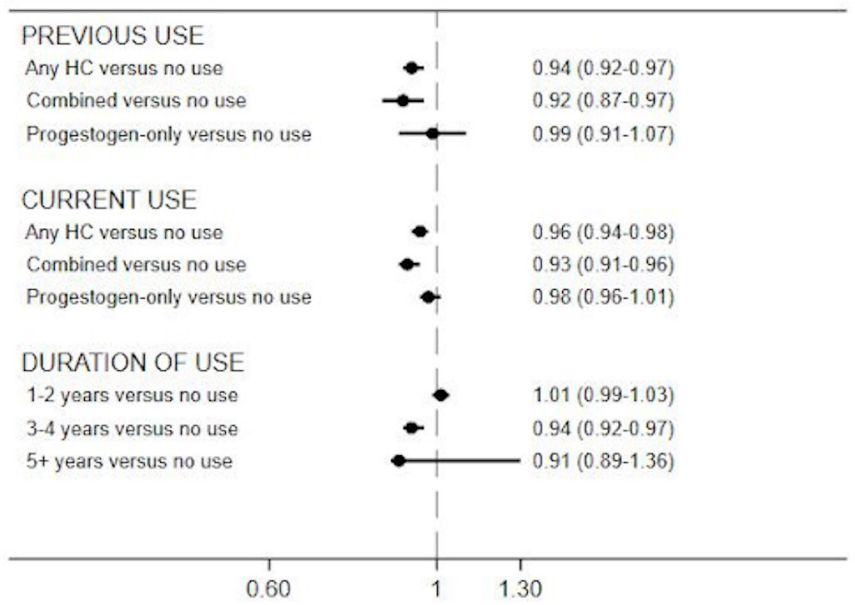

Figure 2 Unadjusted and adjusted associations between use of hormonal contraceptives (HC) and severe asthma exacerbations rates in reproductive-age women.

of confounding by indication, we exhaustively identified a list of conditions for which hormonal contraceptives are used as potential therapies and adjusted for them in our analyses.
The definition of reproductive-age was based only on the age of the women, given that there was no additional information from the database to enhance these definitions; however, the

Table 2 Association between use of hormonal contraceptives and severe asthma exacerbation rates in reproductive-age women and by body mass index and smoking*

\begin{tabular}{|c|c|c|c|c|c|}
\hline \multirow[b]{3}{*}{ Hormonal contraceptive (HC) use } & \multicolumn{3}{|c|}{ Stratified analyses by BMI } & \multirow{2}{*}{\multicolumn{2}{|c|}{$\begin{array}{l}\text { Stratified analyses by smoking status } \\
\text { Hazard ratio }(95 \% \mathrm{Cl})+\S\end{array}$}} \\
\hline & \multicolumn{3}{|c|}{ Incidence rate ratio $(95 \% \mathrm{Cl}) \dagger \ddagger$} & & \\
\hline & $<25 \mathrm{~kg} / \mathrm{m}^{2}$ & $25-29.9 \mathrm{~kg} / \mathrm{m}^{2}$ & $\geq 30 \mathrm{~kg} / \mathrm{m}^{2}$ & Non-smokers & Ever-smokers \\
\hline \multicolumn{6}{|c|}{ Any $\mathrm{HC}$ during any previous or current year } \\
\hline None & 1 & 1 & 1 & 1 & 1 \\
\hline Yes & 0.92 (0.88 to 0.96$)$ & 0.93 (0.88 to 0.98$)$ & 0.96 (0.92 to 1.01$)$ & 0.92 (0.88 to 0.96$)$ & $0.96(0.93$ to 1.00$)$ \\
\hline \multicolumn{6}{|c|}{ Any $\mathrm{HC}$ during current year of asthma exacerbation } \\
\hline None & 1 & 1 & 1 & 1 & \\
\hline Yes & 0.96 (0.93 to 0.99$)$ & 0.98 (0.95 to 1.02$)$ & 0.94 (0.91 to 0.96$)$ & 0.99 (0.96 to 1.02$)$ & $0.94(0.92$ to 0.96$)$ \\
\hline \multicolumn{6}{|c|}{ Type of HC during any previous or current year } \\
\hline None & 1 & 1 & 1 & 1 & 1 \\
\hline Progestogen-only & 0.91 (0.79 to 1.04$)$ & 0.95 (0.81 to 1.10$)$ & 1.16 (1.00 to 1.33$)$ & $1.17(1.02$ to 1.35$)$ & $0.91(0.82$ to 1.01$)$ \\
\hline Combined oestrogen/progestogen & 0.87 (0.80 to 0.95$)$ & 0.85 (0.76 to 0.95 ) & 1.09 (0.98 to 1.21$)$ & 1.01 (0.91 to 1.11$)$ & $0.87(0.81$ to 0.93$)$ \\
\hline \multicolumn{6}{|c|}{ Type of HC during current year of asthma exacerbation } \\
\hline None & 1 & 1 & 1 & 1 & 1 \\
\hline Progestogen-only & 0.98 (0.93 to 1.02$)$ & 1.02 (0.97 to 1.07 ) & 0.97 (0.94 to 1.01$)$ & $0.98(0.94$ to 1.03$)$ & $0.98(0.96$ to 1.01$)$ \\
\hline Combined oestrogen/progestogen & 0.96 (0.92 to 0.99$)$ & 0.95 (0.91 to 1.00$)$ & 0.87 (0.83 to 0.91$)$ & 0.99 (0.96 to 1.03$)$ & 0.89 (0.87 to 0.92$)$ \\
\hline \multicolumn{6}{|l|}{ Duration of use of any HC (years) } \\
\hline 0 & 1 & 1 & 1 & 1 & 1 \\
\hline $1-2$ & 1.03 (0.99 to 1.07$)$ & $0.96(0.92$ to 1.00$)$ & $1.03(0.99$ to 1.06$)$ & $0.99(0.96$ to 1.03$)$ & $1.02(0.99$ to 1.05$)$ \\
\hline $3-4$ & 0.92 (0.88 to 0.97 ) & 1.00 (0.95 to 1.05$)$ & 0.92 (0.88 to 0.96$)$ & 0.95 (0.90 to 0.99$)$ & $0.93(0.90$ to 0.97$)$ \\
\hline $5+$ & 0.93 (0.89 to 0.97$)$ & 0.92 (0.87 to 0.96$)$ & 0.89 (0.86 to 0.93$)$ & $0.98(0.94$ to 1.02$)$ & $0.87(0.84$ to 0.90$)$ \\
\hline \multicolumn{6}{|c|}{$\begin{array}{l}\text { *All analyses were based on multilevel, mixed-effects Poisson regression that accounted for clustering of patients within general practitioner practices. } \\
\text { †Adjusted for follow-up time, level of adherence to asthma medication, age, smoking, Charlson Comorbidity Index, BMI, gravidity, any gynaecological condition and Index of } \\
\text { Multiple Deprivation. } \\
\neq \text { Stratified analyses performed after interaction term between use of } \mathrm{HC} \text { and BMI gave } \mathrm{p}=0.023 \text { for any } \mathrm{HC} \text { and BMI, } \mathrm{p}=0.000 \text { for } \mathrm{HC} \text { types and } \mathrm{BMI} \text { and } \mathrm{p}=0.113 \text { for duration of } \\
\mathrm{HC} \text { and BMI. } \\
\S \text { Stratified analyses performed after interaction term between use of } \mathrm{HC} \text { and smoking gave } \mathrm{p}=0.048 \text { for any } \mathrm{HC} \text { and smoking, } \mathrm{p}=0.107 \text { for } \mathrm{HC} \text { types and smoking, and } \mathrm{p}=0.000 \\
\text { for duration of } \mathrm{HC} \text { and smoking. }\end{array}$} \\
\hline
\end{tabular}


chosen ages reflect the average age of reproductive age in the UK. Beyond this upper age limit of 45 years, women may use hormonal contraceptives for other indications, such as management of abnormal uterine bleeding, a symptom often reflecting ovulatory dysfunction as the menopause transition approaches. While we used information based only on prescription of hormonal contraceptives, it was not possible to determine whether this reflected actual use of the medications, as we did not have data on medication dispensation or actual use. We had planned to perform propensity score analysis as an adjustment strategy for potential confounding factors. ${ }^{15}$ However, we were unable to do this as planned, and instead we performed conventional confounder adjustment by including the confounding factors in the statistical models because we did not find an appropriate algorithm for propensity score analyses within the context of time-varying, multilevel modelling. Based on the values of the calculated E-values for residual confounding, it is possible that residual confounding may still remain after our confounder adjustment and thus may partly account for some of the observed risk estimates. We planned to study the role of routes of administration of hormonal contraceptives (oral, transdermal, subcutaneous, intramuscular or local intrauterine) but this information was poorly and inconsistently recorded in the database, hence we did not undertake this aspect of the analysis. Registering and publishing the protocol before undertaking the analysis enhanced the transparency of our work.

All of the previous studies identified in our earlier systematic review investigating the role of hormonal contraceptives in clinical outcomes of asthma were cross-sectional studies and hence of inferior quality compared with the current study. ${ }^{12}$ Furthermore, the previous studies were limited because assessment of use of hormonal contraceptives and/or asthma outcomes were based on self-reports and in most studies the sample size was small. In the current study we have attempted to overcome these limitations and to clarify the underlying evidence. Overall, our study, by providing the clearest evidence on the role of use of hormonal contraceptives in clinical outcomes of asthma, indicates that previous, current and long-term use of hormonal contraceptives and subtypes were associated with small, reduced clinical effects in reducing asthma exacerbations in women of reproductive age with established asthma.

Oestrogen and progesterone exhibit varying immunological effects including reported enhancement of Th2 and Th17 responses and inhibition of the synthesis of Th1-, Th2- and Th17-associated cytokines, potentially dependent on the concentrations studied. ${ }^{7}{ }^{27}$ Th17 cytokines in particular are associated with severe asthma, and there is evidence that Th17 cells manifest more IL-17A and IL-23R in women with severe asthma than in men. ${ }^{72}$ Increased IL-17A synthesis is linked to female gender, obesity and asthma severity. ${ }^{28}{ }^{29}$ Studies of the effects of hormonal contraceptives directly in patients, including on distinct Th17 subsets, antigen-presenting cells and cytokines that influence IL-17A synthesis, and fatty acid metabolism may provide mechanistic insights beyond those derived from animal models and laboratory studies. ${ }^{30} 31$ Menstruation is accompanied by fluctuations in the levels of oestradiol and progesterone, which seem to worsen asthma symptoms in some women. ${ }^{32}$ It is believed that the use of hormonal contraceptives to suppress these hormonal fluctuations could confer protection from poor asthma outcomes. Both oestrogen and progesterone are known to influence smooth muscle functions, inflammation and airway responsiveness. ${ }^{33}$ While supporting this proposed protective role of hormonal contraceptives on clinical outcomes of asthma, our study demonstrates that the impact on asthma exacerbation may be rather small. Although our findings do not suggest any changes to current asthma management guidelines, they do, however, indicate the need for further mechanistic studies to better understand how hormonal contraceptives influence clinical outcomes in women with established asthma.

Stratification of our results by BMI and smoking status did not reveal any major differences across BMI and smoking categories. The impact of hormonal contraceptives on asthma may be modified by the BMI, given the close interplay between endogenous sex hormones and body fat mass. ${ }^{1134}$ In lean women, endogenous oestrogen is low compared with higher levels in heavier women. ${ }^{35}$ In a cross-sectional study of the association between use of oral contraceptive pills and asthma and allergy in women aged 25-44 years, Macsali and colleagues, by stratifying the results by BMI, found that use of oral contraceptive pills was associated with an increased risk of reported asthma attacks and allergy in normal weight and overweight women but not in lean women. ${ }^{34}$ Besides being a cross-sectional study, contrasting the longitudinal design of the current study, these results observed by Macsali and colleagues ${ }^{34}$ contrast the findings from the current study in which we found a protective effect of hormonal contraceptives on severe asthma exacerbations across all BMI categories. In our previous analysis of the serial cross-sectional Scottish Health Surveys we found that while the risk of asthma care episodes was decreased in overweight or obese women who were using combined oral contraceptives, the risk was increased in overweight and obese women who were not using hormonal contraceptives. ${ }^{11}$ In the current study, the results for combined oestrogen/progestogen contraceptives showed statistical significance across the BMI categories while the results for progestogen-only contraceptives did not. While this may reflect inadequate power to detect a statistical significance for progestogen-only contraceptives, the differences may also reflect the oestrogen effect, given the interplay observed between body mass and local oestrogen production. ${ }^{36}$ Among postmenopausal women, use of menopausal hormone therapy has been linked to greater risk of asthma in lean than in overweight/obese women, and this has been attributed to oestrogen effect. ${ }^{37}{ }^{38}$ Regarding smoking, a potential effect-effect modification is possible, given the suggestion that smoking may alter the haemostasis profiles in oral contraceptive users. ${ }^{39} 40$ While Macsali and colleagues found an increased risk of asthma similarly for women who were smoking and those not smoking, ${ }^{34}$ the findings from the current study showed that use of hormonal contraceptives is associated with a decreased risk of severe asthma exacerbations similarly for non-smokers and ever-smokers.

\section{CONCLUSIONS}

In conclusion, we observed that the use of hormonal contraceptives is associated with a small beneficial effect on severe asthma exacerbation. Further longitudinal studies are required to confirm these findings, as well as mechanistic studies investigating the underlying biological processes through which exogenous sex steroids influence the pathogenesis of asthma in women.

\section{Author affiliations}

${ }^{1}$ Krefting Research Centre, Institute of Medicine, University of Gothenburg, Goteborg, Sweden

${ }^{2}$ Wallenberg Centre for Molecular and Translational Medicine, University of Gothenburg, Gothenburg, Sweden

${ }^{3}$ Asthma UK Centre for Applied Research, Centre for Medical Informatics, Usher Institute, The University of Edinburgh, Edinburgh, UK

${ }^{4}$ Optimum Patient Care, 5 Coles Lane, Cambridge, United Kingdom

${ }^{5}$ Medical Research Council Centre for Reproductive Health, Queen's Medical

Research Institute, The University of Edinburgh, Edinburgh, UK 
${ }^{6}$ Academic Primary Care, Division of Applied Health Sciences, University of Aberdeen, Aberdeen, United Kingdom

${ }^{7}$ Observational and Pragmatic Research Institute, Singapore

${ }^{8}$ Asthma UK Centre in Allergic Mechanisms of Asthma, School of Immunology and Microbial Sciences, Guys Hospital, King's College London, UK, London, UK

${ }^{9} \mathrm{~S} c h$ ool of Health, Wellington Faculty of Health, Victoria University of Wellington, Wellington, New Zealand

${ }^{10}$ Hull York Medical School, Institute for Clinical and Applied Health Research (ICAHR), University of Hull, Hull, UK

\section{Twitter David B Price @OPRI_SG and Aziz Sheikh @DrAzizSheikh}

Acknowledgements The authors would like to thank Dr Lynn Morrice for administrative assistance and members of the Patient and Public Involvement group of the Asthma UK Centre for Applied Research who helped shape this project during the grant application stage. They also thank Dr Susannah McLean who helped with selection of Read codes and design of strategy for determining start and end dates of pregnancies. They are grateful to Optimum Patient Care (OPC) and Observational and Pragmatic Research Institute Pte Ltd (OPRI) for making the OPCRD database ( www.opcrd.co.uk) available free of charge. They also thank Derek Skinner of OPRI who contributed to the creation of the study data-cut, identification of Read codes and Index of Multiple Deprivation (IMD) centiles. BN acknowledges the support of Knut and Alice Wallenberg Foundation, the Wallenberg Centre for Molecular and Translational Medicine, University of Gothenburg, Sweden, and the VBG Group Herman Krefting Foundation on Asthma and Allergy. AS acknowledges the support of Health Data Research UK (BREATHE).

Contributors BIN, AS: conceived idea for the study; BN, AS, CRS, CMH: designed the study; BN, AS, RP, DBP, INS, FA: data collection; BIN, RP, HT, AS, SAS, SM: data analyses; DPR, DBP, SM: clinical/primary care expertise on asthma and OPCRDrelated expertise; HC: expertise on sex steroids; BIN, AS: drafted the manuscript. All authors critically revised the manuscript for intellectual content and approved the final version.

Funding This work was supported by Asthma UK (grant number AUKIG-2016-346) and Health Data Research UK.

Competing interests None declared.

Patient consent for publication Not required.

Provenance and peer review Not commissioned; externally peer reviewed.

Data availability statement The study data are not publicly available as per an agreement with the data custodian.

\section{ORCID iDs}

Bright I Nwaru http://orcid.org/0000-0002-2876-6089

David B Price http://orcid.org/0000-0002-9728-9992

\section{REFERENCES}

1 Osman M, Hansell AL, Simpson CR, et al. Gender-specific presentations for asthma, allergic rhinitis and eczema in primary care. Prim Care Respir J 2007;16:28-35.

2 Prescott E, Lange P, Vestbo J, et al. Effect of gender on hospital admissions for asthma and prevalence of self-reported asthma: a prospective study based on a sample of the general population. Copenhagen City Heart Study Group. Thorax 1997;52:287-9.

3 Real FG, Svanes C, Omenaas ER, et al. Menstrual irregularity and asthma and lung function. J Allergy Clin Immunol 2007;120:557-64.

4 Agarwal SK, Marshall GD. Perimenstrual alterations in type-1/type-2 cytokine balance of normal women. Ann Allergy Asthma Immunol 1999;83:222-8.

5 Siroux V, Curt F, Oryszczyn M-P, et al. Role of gender and hormone-related events on IgE, atopy, and eosinophils in the epidemiological study on the genetics and environment of asthma, bronchial hyperresponsiveness and atopy. J Allergy Clin Immunol 2004:114:491-8.

6 Sánchez-Ramos JL, Pereira-Vega AR, Alvarado-Gómez F, et al. Risk factors for premenstrual asthma: a systematic review and meta-analysis. Expert Rev Respir Med 2017;11:57-72

7 Newcomb DC, Cephus JY, Boswell MG, et al. Estrogen and progesterone decrease let-7f microRNA expression and increase IL-23/L-23 receptor signaling and IL-17A production in patients with severe asthma. J Allergy Clin Immunol 2015;136:1025-34

8 Lange P, Parner J, Prescott E, et al. Exogenous female sex steroid hormones and risk of asthma and asthma-like symptoms: a cross sectional study of the general population. Thorax 2001;56:613-6.

9 Macsali F, Real FG, Omenaas ER, et al. Oral contraception, body mass index, and asthma: a cross-sectional Nordic-Baltic population survey. J Allergy Clin Immunol 2009:123:391-7.

10 Haggerty CL, Ness RB, Kelsey S, et al. The impact of estrogen and progesterone on asthma. Ann Allergy Asthma Immunol 2003:90:284-91.
11 Nwaru BI, Sheikh A. Hormonal contraceptives and asthma in women of reproductive age: analysis of data from serial national Scottish health surveys. J $R$ Soc Med 2015; 108:358-71.

12 McCleary N, Nwaru BI, Nurmatov UB, et al. Endogenous and exogenous sex steroid hormones in asthma and allergy in females: a systematic review and meta-analysis. J Allergy Clin Immunol 2018;141:1510-3.

13 Price DB, Rigazio A, Campbell JD, et al. Blood eosinophil count and prospective annua asthma disease burden: a UK cohort study. Lancet Respir Med 2015:3:849-58.

14 Jones RCM, Price D, Ryan D, et al. Opportunities to diagnose chronic obstructive pulmonary disease in routine care in the UK: a retrospective study of a clinical cohort. Lancet Respir Med 2014;2:267-76.

15 Nwaru BI, Simpson CR, Soyiri IN, et al. Exogenous sex steroid hormones and asthma in females: protocol for a population-based retrospective cohort study using a UK primary care database. BMJ Open 2018;8:e020075.

16 Williams T, van Staa T, Puri S, et al. Recent advances in the utility and use of the General Practice Research Database as an example of a UK Primary Care Data resource. Ther Adv Drug Saf 2012;3:89-99.

17 Charlson ME, Pompei P, Ales KL, et al. A new method of classifying prognostic comorbidity in longitudinal studies: development and validation. J Chronic Dis 1987:40:373-83.

18 Kvaskoff M, Mu F, Terry KL, et al. Endometriosis: a high-risk population for major chronic diseases? Hum Reprod Update 2015;21:500-16.

19 McLennan D, Noble S, Noble M, et al. The English indices of deprivation. London: Ministry of Housing, Communities and Local Government, 2019.

20 Chung KF, Wenzel SE, Brozek JL, et al. International ERS/ATS guidelines on definition, evaluation and treatment of severe asthma. Eur Respir J 2014:43:343-73.

21 Colice G, Chisholm A, Dima AL, et al. Performance of database-derived severe exacerbations and asthma control measures in asthma: responsiveness and predictive utility in a UK primary care database with linked questionnaire data. Pragmat Obs Res 2018;9:29-42.

22 Garson D. Multilevel modelling: applications in STATA $\circledast$, IBM $®$ SPSS $®$, SAS®, R \& HLM. California, USA: Sage Publications, Inc, 2019

23 VanderWeele TJ, Ding P. Sensitivity analysis in observational research: introducing the E-value. Ann Intern Med 2017;167:268-74.

24 von Elm E, Altman DG, Egger M, et al. The Strengthening the Reporting of Observational Studies in Epidemiology (STROBE) Statement: guidelines for reporting observational studies. J Clin Epidemiol 2008:61:344-9.

25 Benchimol El, Smeeth L, Guttmann A, et al. The reporting of studies conducted using observational routinely-collected health data (record) statement. PLoS Med 2015; 12:e1001885

26 Simpson CR, Sheikh A. Trends in the epidemiology of asthma in England: a national study of 333,294 patients. J R Soc Med 2010;103:98-106.

27 Piccinni M-P, Lombardelli L, Logiodice F, et al. Medroxyprogesterone acetate decreases Th1, Th17, and increases Th22 responses via AhR signaling which could affect susceptibility to infections and inflammatory disease. Front Immunol 2019;10:642.

28 Fuseini H, Newcomb DC. Mechanisms driving gender differences in asthma. Curr Allergy Asthma Rep 2017;17:19.

29 Endo Y, Yokote K, Nakayama T. The obesity-related pathology and Th17 cells. Cell Mol Life Sci 2017;74:1231-45.

30 Bettelli E, Carrier Y, Gao W, et al. Reciprocal developmental pathways for the generation of pathogenic effector Th17 and regulatory T cells. Nature 2006;441:235-8

31 Berod L, Friedrich C, Nandan A, et al. De novo fatty acid synthesis controls the fate between regulatory T and T helper 17 cells. Nat Med 2014;20:1327-33.

32 Bonds RS, Midoro-Horiuti T. Estrogen effects in allergy and asthma. Curr Opin Allergy Clin Immunol 2013;13:92-9

33 Haggerty CL, Ness RB, Kelsey S, et al. The impact of estrogen and progesterone on asthma. Ann Allergy Asthma Immunol 2003;90:284-91.

34 Macsali F, Real FG, Plana E, et al. Early age at menarche, lung function, and adult asthma. Am J Respir Crit Care Med 2011;183:8-14.

35 Green LE, Dinh TA, Smith RA. An estrogen model: the relationship between body mass index, menopausal status, estrogen replacement therapy, and breast cancer risk. Comput Math Methods Med 2012;2012:1-8.

36 Kalish GM, Barrett-Connor E, Laughlin GA, et al. Association of endogenous sex hormones and insulin resistance among postmenopausal women: results from the Postmenopausal Estrogen/Progestin Intervention Trial. J Clin Endocrinol Metab 2003;88:1646-52.

37 Gómez Real F, Svanes C, Björnsson EH, et al. Hormone replacement therapy, body mass index and asthma in perimenopausal women: a cross sectional survey. Thorax 2006:61:34-40.

38 Jarvis $\mathrm{D}$, Leynaert $\mathrm{B}$. The association of asthma, atopy and lung function with hormone replacement therapy and surgical cessation of menstruation in a population-based sample of English women. Allergy 2008;63:95-102.

39 Fruzzetti F. Hemostatic effects of smoking and oral contraceptive use. Am J Obstet Gynecol 1999;180:S369-74.

40 Fruzzetti F, Ricci C, Fioretti P. Haemostasis profile in smoking and nonsmoking women taking low-dose oral contraceptives. Contraception 1994:49:579-92. 